a copper surface. They observed that the Raman spectra obtained are different when the tip of the microscope is placed above different regions of the molecule. By plotting the map of Raman intensities for a given vibrational energy, the authors revealed the locations of the atoms most involved in the motion of the associated normal mode. In other words, they obtained snapshots of each vibrational mode (Fig. 1).

These experiments raise questions regarding the mechanisms that enable such a high resolution to be achieved ${ }^{9}$. Hotspots are normally no smaller than nanoscale sizes, which makes it difficult to explain how resolution at much smaller scales can be obtained. Moreover, the electromagnetic theory of continuous media, which explains surface-enhanced Raman scattering, breaks down at the subnanometre scales at which non-local and quantum effects come into play. Full quantum-chemical models of molecules sandwiched between a metal surface and a microscope tip might help us understand how ångström-scale resolution is achieved.
There are limitations to the general use of Lee and colleagues' approach. The experiments are carried out in an ultrahigh vacuum and at a very low temperature (6 kelvin), but such conditions are not accessible to all scientists. Other issues are that only certain pairs of molecules and substrates will provide sufficient anchoring to reach ångström-scale resolution, and the vibrational modes of the molecules might be affected by the substrate. Moreover, the technique is mainly sensitive to motion that is perpendicular to the substrate, rather than to parallel motion.

Nevertheless, overcoming these limitations could open up exciting opportunities, for example in the imaging of biomolecules. One can also envisage combining this ultrahigh-resolution method with approaches that enable ultrafast TERS measurements ${ }^{10}$, to record movies of vibrating molecules. But for now, just being able to experimentally image a vibrational mode will no doubt delight many spectroscopists, who have previously had to rely on theoretical predictions and visualizations.
Eric C. Le Ru is at the MacDiarmid Institute for Advanced Materials and Nanotechnology, School of Chemical and Physical Sciences, Victoria University of Wellington, Wellington 6140, New Zealand. e-mail:eric.leru@vuw.ac.nz

1. Steidtner, J. \& Pettinger, B. Phys. Rev. Lett. 100, 236101 (2008)

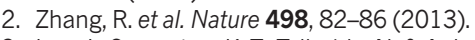

3. Lee, J., Crampton, K. T., Tallarida, N. \& Apkarian, V. A. Nature 568, 78-82 (2019).

4. Le Ru, E. C. \& Etchegoin, P. G. Surface-Enhanced Raman Spectroscopy and Related Plasmonic Effects (Elsevier, 2009).

5. Sonntag, M. D. et al. J. Phys. Chem. C 116, 478-483 (2012).

6. Le Ru, E. C. \& Etchegoin, P. G. Annu. Rev. Phys. Chem. 63, 65-87 (2012).

7. Stöckle, R. M., Suh, Y. D., Deckert, V. \& Zenobi, R. Chem. Phys. Lett. 318, 131-136 (2000).

8. Pettinger, B., Ren, B., Picardi, G., Schuster, R. \& Ertl, G. Phys. Rev. Lett. 92, 096101 (2004).

9. Duan, S. et al. J. Am. Chem. Soc. 137, 9515-9518 (2015).

10.Pozzi, E. A. et al. J. Phys. Chem. Lett. 5, 2657-2661 (2014).

\section{New interactions seen in an ultracold gas}

Another A atom would be attracted to this absence of $B$ atoms, and the end result would again be an attractive mediated interaction between the $\mathrm{A}$ atoms. This simple argument explains why mediated interactions are inherently attractive at short distances. At longer distances, the story is more complicated, because intricate quantum effects can make a mediated interaction repulsive.

DeSalvo and colleagues used a mixture of bosonic caesium-133 atoms and fermionic lithium-6 atoms - bosons have integer spin angular momentum, whereas fermions have half-integer spin. The atoms were confined near the minimum of a harmonic trap, which is a type of potential-energy surface (often simply called a potential) that has a parabolic form. The authors cooled the mixture to extremely
$\mathrm{M}$ any of us have sat in a boat that is being rocked by the waves from another passing vessel. This situation is an example of a mediated interaction: even though the two boats do not touch, they interact through the exchange of waves in the water between them. Mediated interactions have a central role in modern physics. For instance, the fundamental interactions between elementary particles are mediated by particles such as photons ${ }^{1}$. And the attraction between electrons that leads to superconductivity in metals arises from the exchange of vibrations in the surrounding crystal lattice ${ }^{2}$. On page 61 , DeSalvo et al. ${ }^{3}$ report the first observation of mediated interactions in an ultracold mixture of lithium and caesium atoms (Fig. 1a).

Consider a mixture of two kinds of atom, $\mathrm{A}$ and $\mathrm{B}$, and suppose, for clarity, that there are many more $B$ atoms than there are $A$ atoms. Let us first assume that the $\mathrm{A}$ and $\mathrm{B}$ atoms attract each other. Because of this attraction, there would be a high density of B atoms around a given $\mathrm{A}$ atom (Fig. 1b). Another $\mathrm{A}$ atom would be attracted to these $\mathrm{B}$ atoms, and the net effect would be an attractive interaction between the
A atoms that is mediated by the B atoms.

Had we instead assumed that the two kinds of atom repel each other, there would be a low density of B atoms around a given A atom.

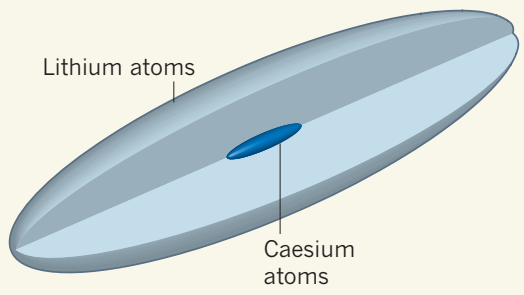

b

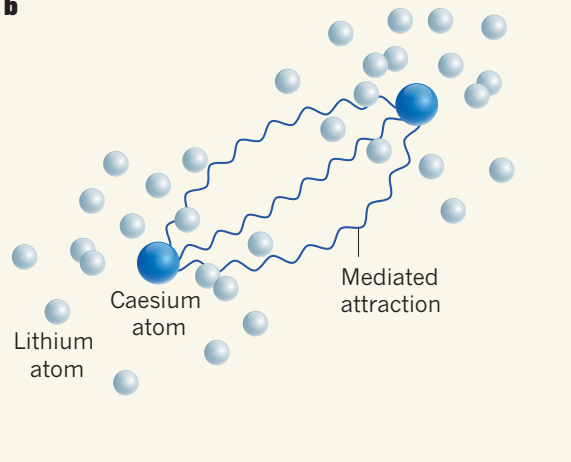

Figure 1 | An ultracold mixture of lithium and caesium atoms. a, DeSalvo et al. ${ }^{3}$ report an experiment in which caesium atoms in a state of matter known a Bose-Einstein condensate are surrounded by lithium atoms in the form of a quantum gas called a Fermi gas. $\mathbf{b}$, When there is an attractive interaction between the two types of atom, there is a high density of lithium atoms around a given caesium atom. Another caesium atom is attracted to this concentration of lithium atoms, resulting in an attractive interaction between the caesium atoms that is mediated by the lithium atoms. A similar argument shows that a repulsive interaction between the two kinds of atom also leads to an attractive mediated interaction between the caesium atoms (not shown). 
low temperatures, so that the caesium atoms formed an exotic state of matter known as a Bose-Einstein condensate (BEC), whereas the lithium atoms instead formed a quantum gas called a Fermi gas.

The direct interaction between the caesium atoms was weakly repulsive, whereas the direct interaction between the caesium and lithium atoms could be tuned to be either attractive or repulsive. There was no direct interaction between the lithium atoms because of a fundamental principle of quantum mechanics that states that two fermions cannot occupy the same space. This principle also meant that the lithium gas had a much larger volume than the BEC of caesium atoms, which was located at the centre of the gas. DeSalvo et al. demonstrated that the presence of the lithium gas had two effects on the caesium BEC.

First, when the BEC was set to oscillate back and forth in the harmonic trap, the oscillation frequency changed when the lithium gas was present. The reason for this change is that the energy of the caesium atoms was shifted by the direct interaction between the caesium and lithium atoms. This energy shift was spatially dependent because the density of the lithium gas decreased as a function of distance from the centre of the trap. As a result, the BEC was subjected to an additional trapping potential from the lithium gas that altered the oscillation frequency.

The second, and arguably more intriguing, effect was that the lithium gas made the BEC decrease in size. By carefully analysing their experimental data, DeSalvo and colleagues concluded that this effect was caused by an attractive interaction between the caesium atoms that was mediated by the lithium gas. The results were in fair agreement with theoretical predictions based on a type of interaction called a Ruderman-Kittel-Kasuya-Yosida (RKKY) interaction, which has been shown ${ }^{4-6}$ to exist as an interaction mediated by a Fermi gas precisely like the lithium gas.

RKKY interactions give rise to a great variety of magnetic phenomena in rare-earth elements - the collective name for 17 chemically similar metallic elements - in which electrons play the part of the Fermi gas. In addition, electronic devices, such as hard drives, that exploit a phenomenon known as the giant-magnetoresistance effect contain magnetic layers that are thought to be coupled by RKKY-like interactions .

Although the observed mediated interaction between the caesium atoms was quite weak, DeSalvo et al. used a clever trick to show that it could still have spectacular effects. The authors tuned the direct interaction between the caesium atoms to be only very weakly repulsive so that the attractive mediated interaction was comparatively stronger. The combination of the direct and mediated interactions then gave rise to a net attraction between the caesium atoms. Because, unlike for fermions, nothing prevents bosons from occupying the same space, the BEC collapsed. The authors observed this collapse through the formation of small, soliton-like blobs of caesium atoms solitons are spatially localized states that are characteristic of BECs.

Given the unrivalled versatility of atomic gases, DeSalvo and colleagues' results open up the possibility of exploring mediated interactions in detail and probing interactions that have never been seen before. So far, only a weak mediated interaction has been observed, and it would be useful to study stronger interactions. Such interactions should greatly affect the energy spectrum of excitations of the $\mathrm{BEC}^{8}$ and give rise to a range of exotic phases of matter in mixtures of bosons and fermions $^{9,10}$.

Future work should also explore the reciprocal case of an interaction between fermions that is mediated by a BEC. This mediated interaction is, in general, much stronger than the fermion-mediated interaction because of the large compressibility of a BEC compared with a Fermi gas, and it could also lead to previously unobserved phases of matter ${ }^{11-13}$. It will be exciting to see what discoveries follow the breakthrough results of DeSalvo and colleagues.

Georg M. Bruun is in the Department of Physics and Astronomy, Aarhus University, Aarhus 8000, Denmark.

e-mail:bruungmb@phys.au.dk

1. Weinberg, S. The Quantum Theory of Fields (Cambridge Univ. Press, 1995).

2. Schrieffer, J. R. Theory of Superconductivity (Perseus, 1999).

3. DeSalvo, B. J., Patel, K., Cai, G. \& Chin, C. Nature 568, 61-64 (2019).

4. Ruderman, M. A. \& Kittel, C. Phys. Rev. 96, 99-102 (1954).

5. Kasuya, T. Prog. Theor. Phys. 16, 45-57 (1956).

6. Yosida, K. Phys. Rev. 106, 893-898 (1957).

7. Grosso, G. \& Parravicini, G. P. Solid State Physics 2nd edn (Elsevier, 2013).

8. Kinnunen, J. J. \& Bruun, G. M. Phys. Rev. A 91 041605 (2015).

9. Büchler, H. P. \& Blatter, G. Phys. Rev. Lett. 91, 130404 (2003)

10.Sachdev, S. Nature Phys. 4, 173-185 (2008).

11. Heiselberg, H., Pethick, C. J., Smith, H. \& Viverit, L. Phys. Rev. Lett. 85, 2418-2421 (2000).

12.Bijlsma, M. J., Heringa, B. A. \& Stoof, H. T. C. Phys. Rev. A 61, 053601 (2000)

13.Camacho-Guardian, A. \& Bruun, G. M. Phys. Rev. X 8, 031042 (2018).

\section{BIODIVERSITY}

\section{A mountain of ecological interactions}

A detailed biological assessment of Africa's highest mountain explores how climate modulates the effects of human land use on plants, animals, microorganisms and a diverse array of ecosystem functions. SEE LETTER P.88

\section{ROBERT M. PRINGLE}

$\mathrm{M}$ ountains support roughly one-third of all land-dwelling species and supply water for nearly half of all people $^{1}$. The ecology of mountain environments is strongly influenced by climate $e^{2-4}$. For example, because temperatures drop as altitude increases, organisms that have greater cold tolerance are favoured at higher elevations. Accordingly, there is a rapid change in the species present as one moves up a mountain from the warm lowlands to the cold highlands. And because few organisms can withstand the most-extreme conditions, the total number of species tends to be low on mountaintops.

Climate change is now rearranging the pieces of this puzzle ${ }^{5,6}$, and ecologists are struggling to predict the picture that will emerge $e^{7,8}$. One major source of uncertainty is the extent to which the effects of human land use (activities such as farming and logging) might interact with climatic factors to shape the distribution of species and the operation of biogeochemical processes. On page 88, Peters et al. ${ }^{9}$ report their analysis of an astonishingly comprehensive ecological data set from Mount Kilimanjaro (Fig. 1), which shows that temperature and rainfall modulate the effects of human land use on biodiversity and ecosystems.

A previous study ${ }^{10}$ from the same research group revealed that the number of plant and animal species declines at an almost linear rate as elevation increases on Mount Kilimanjaro, suggesting that temperature is the main determinant of species richness. Peters et al. have expanded the scope and scale of that earlier assessment. Their new study reports data gathered over 6 years by 50 researchers at 60 sites ranging from 866 to 4,550 metres above sea level. These sites represented both natural habitats, such as lowland savannahs and alpine heaths, and habitats that had been heavily affected by human activity, including cropland and logged forests. The authors noted the number of species of plants, animals and soil-dwelling bacteria at each study site. They also recorded data for 30 different ecosystem functions, which are processes related to the transfer of energy and matter through 